\title{
EL CLIMA LABORAL COMO UN ELEMENTO DEL COMPROMISO ORGANIZACIONAL*
}

\author{
LUIS ROBERTO DOMÍNGUEZ AGUIRRE \\ Instituto Tecnológico Superior de Puerto Vallarta, México \\ luisrda@hotmail.com \\ ÁLVARO FABRICIO RAMÍREZ CAMPOS \\ Instituto Tecnológico Superior de Puerto Vallarta, México \\ a_branches@hotmail.com \\ ANDRÉS GARCÍA MÉNDEZ \\ Centro Universitario de la Costa de la Universidad de Guadalajara, México \\ angame_97@hotmail.com
}

\section{RESUMEN}

El compromiso y el clima organizacional son actitudes que reflejan un estado psicológico relacionado con los valores y objetivos de una organización. Existe un conjunto de causales para que una persona o un conjunto de personas sostengan un compromiso en las organizaciones, teóricamente la percepción favorable del clima laboral que les rodea incrementa el compromiso. El estudio muestra las relaciones observadas en las dimensiones del clima organizacional y las del compromiso. Los hallazgos señalan que tres de los cuatro factores en que se agrupan las dimensiones del clima organizacional se correlacionan significativamente con el compromiso institucional.

PALABRAS CLAVE: CLIMA ORGANIZACIONAL, COMPROMISO, COMPORTAMIENTO.

\section{ABSTRACT}

Commitment and organizational climate are attitudes that reflex a psychological state related to the values and objectives of an organization. There is a set of causes for a person or a group to make commitments

* $\quad$ El presente artículo es producto del Proyecto proyecto "El clima Organizacional organizacional y sus relaciones con variables de resultados" que, se trabaja en el Instituto Tecnológico Superior de Puerto Vallarta en su primera fase, inicia en y que inició en agosto de 2011 y que tiene su término eny se proyecta hasta diciembre de 2012. in organizations and, theoretically, their perception of a favorable labor climate around them increases those commitments. This article shows the relationships between organizational climate and commitment. The findings show that organizational climate dimensions significantly correlate to institutional commitment.

KEY WORDS: ORGANIZATIONAL CLIMATE, COMMITMENT, BEHAVIOR.

\section{INTRODUCCIÓN}

El entorno económico actual, los cambios tecnológicos y sociales derivados del crecimiento exponencial del conocimiento y la búsqueda constante de elementos competitivos han hecho que la administración de personas en una organización o institución sea más complicada, se entiende así que existe una relación positiva entre la eficacia de estas personas y el logro de objetivos organizacionales.

Todos los recursos son importantes en una organización, pero los recursos financieros, tecnológicos, materiales e incluso los comerciales pueden llegar a ser competitivos. Son las personas las que pueden hacer la diferencia en una organización. Las habilidades, el entusiasmo, la satisfacción en el trabajo y la lealtad hacia la organización de los trabajadores influyen sobre los resultados, la cali- 
dad, la productividad, la eficiencia, la imagen, la utilidad, la rentabilidad y la competitividad de la organización.

La complicación en el manejo de una comunidad laboral no se observa solo en organizaciones empresariales, sino también en instituciones de educación superior (IES), en las últimas décadas se ha manifestado un incremento vertiginoso de la matrícula en las IES (Tuiran, 2011), mientras su situación financiera permanentemente deficitaria ha hecho surgir una amplia variedad de situaciones y problemas. Son familiares los diagnósticos que señalan la problemática de la calidad de la docencia, la investigación y la difusión cultural que ofrecen los centros educativos de nivel superior (Marúm-Espinoza, 2009). No obstante, resultan menos frecuentes, o poco difundidos, aquellos relativos a las circunstancias prevalecientes en los procesos administrativos que apoyan a estas funciones; aunque son reconocidos ciertos problemas, producto del crecimiento y desarrollo desequilibrado de estas instituciones.

Algunos de los inconvenientes citados son el crecimiento excesivo de las áreas administrativas, un funcionamiento burocrático y procesos obsoletos, la falta de profesionalización del personal técnico administrativo, la rigidez en el control de gestión y la multiplicación de áreas con funciones similares. En ocasiones, también se concentra el poder de decisión en instancias de la administración central como efecto del abandono y desinterés de la comunidad académica, existe un uso inadecuado de herramientas tecnológicas de alto costo y gran potencial, y distorsiones operativas creadas por la insuficiencia de los apoyos financieros o por la falta de oportunidad en su entrega. Es frecuente, además, la carencia de procesos de control y seguimiento operativo que hagan posible una evaluación sistemática, rigurosa y confiable de las actividades administrativas.
A lo largo de los últimos años, se han propuesto acciones tendientes a mejorar las tareas administrativas. Así, el Programa Integral para el Desarrollo de la Educación Superior (PROIDES) impulsó cuatro proyectos para determinar las causas y proponer soluciones a los problemas administrativos de mayor relevancia, los cuales estuvieron relacionados con la formación del personal administrativo, los aspectos presupuestarios y financieros, la organización académico-administrativa y los aspectos normativos.

Aunque los avances en los proyectos citados fueron limitados, han permitido comprobar que en la administración de las IES se presenta una problemática que comprende las áreas consideradas tradicionalmente en la función de apoyo administrativo y la administración del quehacer académico. Pocas veces se considera que son componentes de la gestión académica las tareas de carácter administrativo que propician la conducción, por una vía institucional, de los procesos de docencia, investigación y difusión y extensión, los que en última instancia son responsabilidad de los funcionarios de las áreas académicas (ANUIES).

El reto actual de la dirección de una institución de educación superior radica en crear herramientas útiles mediante las cuales su comunidad pueda llegar a comprometerse con los objetivos institucionales e integrarse en su proyecto estratégico.

Las ventajas que consiguen las organizaciones con sus recursos y capacidades serán válidas si pueden ser mantenidas en el tiempo, lo cual dependerá de la durabilidad de los recursos, de su movilidad y de la posibilidad de imitarlos. En lo referente a los recursos humanos, su duración depende de la permanencia de los individuos en la organización, que estará relacionada con el grado de satisfacción que su trabajo les reporte en todos los niveles. Por otro lado, las habilidades conjuntas como miembros de equipos de 
trabajo tienden a perdurar e incluso a mejorar a través de la experiencia.

El compromiso organizacional es uno de los mecanismos que posee la dirección de una institución para analizar la lealtad y vinculación de los empleados con su organización, la cual existe entre los miembros de una organización, cuando las personas se identifican con la misma, o cuando los objetivos de la organización y los objetivos individuales están muy integrados y son totalmente congruentes.

De esta forma, si se consigue que los empleados estén muy identificados e implicados en la organización en la que trabajan, mayores serán las probabilidades de que permanezcan en la misma, posibilitando el desarrollo de las habilidades y capacidades colectivas y formando equipos de trabajo cohesionados, que en definitiva conducirán a que la organización sea más eficiente y logre sus objetivos. Por otra parte, se buscará que los individuos se impliquen en la organización con base en sus propios intereses; si los costos de abandonar la organización son muy elevados debido a políticas salariales, incentivos, relaciones, prestigio u otras políticas de gestión de recursos humanos, los individuos tienden a permanecer en ella manteniéndose de nuevo las capacidades colectivas dentro de la misma, como sustento de las ventajas sostenibles y duraderas que la organización sería capaz de conseguir.

La importancia del compromiso organizacional radica en que muestra un impacto directo en las actitudes y conductas de los empleados, tales como aceptación de metas, valores y cultura de la organización, menor ausentismo y baja rotación de personal entre otras (Betanzos Díaz y Paz Rodríguez, 2007).

El compromiso organizacional toma una estructura orientada al cumplimiento de la misión y visión de la organización y sus beneficios, si los empleados no se encuentren comprometidos se produce una disminución en la eficacia de la organización (Soberanes y De la Fuente, 2009), la cual favorece el uso irracional de los recursos y en va en detrimento de la eficiencia. Por otro lado, una comunidad comprometida fortalece la competitividad de las organizaciones, dado que existe un decrecimiento en los índices de rotación de personal y de los gastos derivados del reclutamiento, selección y capacitación en la sustitución de personal.

Esta relación simbiótica entre los colaboradores y la organización, tomando en consideración que el éxito en las organizaciones para mantener su permanencia en el mercado, depende no solo del o los directivos, sino también en gran medida de la implicación positiva e integración adecuada de sus colaboradores (Etzioni, 1975), realza la necesidad de desarrollar capital humano y mantener la inversión dentro de la institución para aprovechar los conocimientos y el capital intelectual de sus integrantes (Hall, 1996). Sin embargo, para que ésta situación se manifieste en las organizaciones es necesario contar con la participación de los colaboradores más que con la intención y deseos de los directivos, por ello es de suma relevancia integrar a los colaboradores con un compromiso organizacional elevado.

\section{El compromiso organizacional}

Existe una amplia variedad de definiciones y medidas de compromiso organizacional, todas llevan implícitas componentes afectivos, normativos y calculados. Se parte de las premisas de que el compromiso se manifiesta por el deseo de las personas de estar y continuar en la organización por una causa sentimental (Buchanan, 1974); o por el conjunto de normas políticas y procedimientos que obligan a las personas a continuar dentro de la organización o de realizar un esfuerzo adicional; también la preferencia ante la com- 
paración hecha entre los beneficios ofrecidos por otras organizaciones (Meyer y Allen, 1997).

Otros autores definen el compromiso organizacional en términos de actitudes, como un fuerte deseo de seguir siendo miembro de una organización en particular y una disposición por realizar un esfuerzo extraordinario en beneficio de ella y un reconocimiento firme de los valores y las metas de la organización, así como la aceptación de estos (Mowday, Porter, y Steers, 1982).

El compromiso organizacional es un estado psicológico (Bayona, Goñi, y Madorran, 2000) y todo parece indicar que las diferentes medidas y definiciones del término tienen en común el considerarlo como un vínculo o lazo del individuo con la organización. En otras palabras, es una actitud que refleja la lealtad de los empleados a su organización y es un proceso continuo mediante el cual los participantes organizacionales expresan su preocupación por la organización, su éxito y bienestar continuo.

\section{El clima organizacional}

El clima organizacional se basa en las percepciones individuales, a menudo se define como los patrones recurrentes de comportamiento, actitudes y sentimientos que caracterizan la vida en la organización, y se refieren a las situaciones actuales en una organización y los vínculos entre los grupos de trabajo, los empleados y el desempeño laboral. Por lo tanto, casi siempre la gerencia manipula el clima con mayor facilidad que la cultura, con el fin de afectar de manera directa el comportamiento de los empleados.

No se pretende agotar todas las definiciones que existen del clima organizacional, sino recuperan aquellas que ofrezcan un acercamiento al objeto de estudio desde el ámbito educativo y que reflejen la posición teórica del presente trabajo. De acuerdo con Brunet (2004), el concepto de clima organizacional fue introducido por primera vez en la psicología industrial por Germman, en el año 1960.

Tagiuri y Litwin, citados por Denison (1991), expresan que: "El clima organizacional es una cualidad relativamente permanente del ambiente interno de una organización que: (a) experimentan sus miembros, (b) influye en su comportamiento y (c) se puede describir en función de los valores de un conjunto particular de características (o actitudes) de la organización".

Algunos otros (Martin y Colbs, 1999) lo definen como un concepto metafórico derivado de la metodología, que adquiere relevancia en el ámbito social cuando las empresas comienzan a otorgar importancia a los aspectos relacionados con un ambiente de trabajo satisfactorio para conseguir una mayor producción en términos cuantitativos y cualitativos.

Al respecto Gairín (1996) argumenta que las relaciones que existen entre los miembros de una organización y el conocimiento mutuo que poseen juegan un papel importante en la configuración de la percepción del clima que se genere en el colectivo. Los climas no deben considerarse como estilos cognitivos o mapas lógicos, sino esquemas colectivos de significado.

El clima organizacional es una propiedad del ambiente percibido o experimentado por los miembros de la organización (Chiavenato y Colbs, 2001), esta definición aporta un elemento que ayuda a comprender que el clima organizacional en esencia nos remite a la percepción de los que integran una organización, por lo incluye distintas fuentes de información de los integrantes de una institución.

Goncalves (2000) menciona que el clima organizacional dentro de las instituciones se refleja en las estructuras organizacionales, tamaño de la organización, modos de comunicación y estilo de liderazgo. Los dos últimos elementos, de acuerdo con Brunet (2004), se ubican en los pro- 
cesos organizacionales; no obstante se reconoce que las estructuras organizacionales poseen un peso específico en la percepción de las personas, por ello estos procesos le conceden el carácter de un fenómeno complejo con múltiples variables por investigar.

En síntesis, el clima es el conjunto de percepciones globales que los individuos tienen de su ambiente y que reflejan la interacción entre las características personales del individuo y las de la organización. Al ser una función institucional que se crea, se puede intervenir sobre él. No es sólo un concepto o un fenómeno cuyo conocimiento nos ayudará a entender mejor el funcionamiento de las organizaciones, sino un concepto de intervención que permite la mejora de los resultados organizacionales (Domínguez, Sánchez y Torres, 2010).

En éste orden de ideas, es importante identificar la relación de dependencia existente entre el clima laboral imperante y el compromiso de los colaboradores para así poder identificar si los beneficios que proporciona tanto el clima laboral favorable como un alto compromiso muestra aplicación en la cultura de trabajo en las instituciones de educación superior mexicanas; identificar en cuál grado o nivel y, en consecuencia, contar con los elementos y conocimientos necesarios para proporcionar opciones de intervención que formulen un clima laboral adecuado para el crecimiento del compromiso.

Debido a lo anterior, se plantea en el presente estudio el objetivo de estudiar el compromiso organizacional como herramienta de gestión de recursos humanos, aplicado al caso de un grupo de instituciones públicas de educación superior. Para ello, se agrupó a los individuos en función de su trabajo administrativo para analizar como las variables de clima y compromiso se relacionan entre sí. De esta forma, lo que se pretende es que la institución pueda disponer de un mayor conocimiento de las actitudes de los indivi- duos y así poder incentivar comportamientos que repercutan en mejores resultados.

La satisfacción laboral ha recibido la mayor atención en cuanto a las actitudes relacionadas con el trabajo. Sin embargo, el compromiso organizacional obtiene cada vez mayor reconocimiento en la literatura sobre el comportamiento organizacional. En tanto que la satisfacción laboral se relaciona principalmente con la actitud del empleado hacia el trabajo y el compromiso organizacional lo hace a nivel de la organización, se ha encontrado una fuerte relación entre la satisfacción laboral y el compromiso organizacional con el paso del tiempo (Luthans, 2008).

La misma relación entre compromiso y satisfacción habrá de establecerse con el clima organizacional. Según Luthans (2008) existen individuos satisfechos con sus empleos, pero que detestan la organización altamente burocrática para la que trabajan; o el ingeniero de software puede estar insatisfecho con su empleo actual, pero muy comprometido con la empresa visionaria de alta tecnología.

Se espera observar la relación de las variables independientes sobre las variables dependientes y que posiblemente:

a) No existe relación entre la importancia relativa que los colaboradores de las instituciones de educación superior otorgan al clima, en sus dimensiones de liderazgo, motivación, comunicación y estructura, y los niveles de compromiso en sus dimensiones de afectivo, calculado y normativo.'

$$
R c_{c}=0
$$

b) Existe relación entre la importancia relativa que los colaboradores de las instituciones

1. Rc: Relación múltiple entre clima y compromiso organizacional que puede ir de 0 a 1 o de 0 a - 1 
de educación superior dan al clima, en sus dimensiones de liderazgo, motivación, comunicación y estructura, y sus niveles de compromiso en sus dimensiones de afectivo, calculado y normativo.

\section{$R c_{c} \neq 0$}

\section{METODOLOGÍA}

El estudio fue de corte transversal con un enfoque de tipo cuantitativo y de alcance correlacional. La investigación se llevó a cabo en las instituciones de educación superior de Puerto Vallarta. El total de instituciones que conforman el universo y sobre las que se formuló el diseño del modelo de relaciones suman un total de cinco instituciones con un mínimo de 12 empleados administrativos y un máximo de 261.

El procedimiento de muestreo fue aleatorio y estratificado, se homogeneizaron los estratos para hacer más precisas las estimaciones. Se buscó asegurar que la muestra representara adecuadamente la población, aprovechando que se conocía su distribución se ponderó la muestra con pesos específicos similares a los de la población.

Para medir el clima organizacional y el compromiso, se utilizó un cuestionario que se conformó mediante siete dimensiones que contienen 20 variables del clima organizacional y 15 del compromiso, además de ocho variables de tipo demográfico.

El instrumento, formado de siete dimensiones, con un total de 35 variables, mediante las que se describirían los hechos que ya habían ocurrido en las IES, cumplió con los criterios de validez y confiabilidad, por lo que se desarrolla un análisis sobre la información obtenida con un nivel de seguridad aceptable.
TABLA 1

\section{DIMENSIONES Y NÚMERO DE VARIABLES}

\begin{tabular}{|lc|}
\hline \multicolumn{2}{|c|}{ CLIMA ORGANIZACIONAL } \\
\hline \multicolumn{1}{|c|}{ DIMENSIONES } & No. DEVARIABLES \\
\hline Estructura & 5 \\
Comunicación & 5 \\
Motivación & 5 \\
Liderazgo & 5 \\
TOTAL & 20 \\
\hline & COMPROMISO ORGANIZACIONAL \\
Afectivo & 5 \\
Normativo & 5 \\
Calculado & 5 \\
\hline TOTAL & 15 \\
\hline GRAN TOTAL & 35 \\
\hline
\end{tabular}

Fuente: Elaboración propia

Se aplicaron 384 cuestionarios a personal administrativo de las instituciones de educación superior en Puerto Vallarta, que fue seleccionado de forma aleatoria.

El tamaño de la muestra cumplió con los criterios metodológicos necesarios para 95\% de confianza con +/- 10\% de error. Se distribuyó la muestra de forma proporcional al número de empleados administrativos existentes en cada institución.

La muestra para probar el instrumento la conformaron 145 personas, vinculadas laboralmente a empresas e instituciones en Puerto Vallarta.

La confiabilidad del instrumento se evaluó a través de la técnica de mitades partidas (splithalves), así se hizo una sola aplicación del instrumento dividido en dos y se correlacionaron las dos partes con el programa estadístico SPSS con el índice de correlación $\mathbf{r}$ de Pearson entre ítem- ítem. Luego, se calculó el coeficiente alfa de Cronbach en los distintos componentes del 
clima, reflejando el grado que cobrarían los ítems que constituyen el test, como indicador de la consistencia interna.

Los índices de correlación obtenidos en los 35 ítems demuestran significancia estadística del 0,01 donde solo una pregunta no alcanza relevancia con un índice de relación de 0,15.

Con el fin de comprobar que los componentes del instrumento se agrupaban en dimensiones, se derivaron los factores y se valoró el ajuste global, la información relativa a los 35 posibles factores y su poder explicativo relativo expresado por sus autovalores. Además de valorar la importancia de cada componente, se pudieron emplear los autovalores como ayuda para seleccionar el número de factores.

También se aplicó el criterio de la raíz latente, que consiste en seleccionar los factores bajo el criterio o la racionalidad de que cualquier factor individual deberá justificar la varianza de por lo menos una única variable, cada variable contribuye con un valor de 1 para el autovalor total, por tanto se consideraron los factores que tienen raíces latentes o autovalores mayores que 1. Bajo este criterio, se observaron siete componentes, estos siete componentes representan $62,73 \%$ de la varianza.

El porcentaje para la solución factorial muestra que $62,73 \%$ del total de la varianza está representado por la información contenida en la matriz de la solución para los siete factores. Por tanto, el índice para esta solución está ligeramente más elevado que el umbral normalmente aceptado por las ciencias sociales que es de $60 \%$, aunque no existen acuerdos absolutos con respecto a este umbral, que en algunas ciencias como las naturales se fija en por lo menos 95\% (Hair, Anderson, Tatham, y Black, 2007, p. 93).

Por otra parte, se evaluó el alfa en cada una de las dimensiones del clima organizacional y del compromiso. En el caso del clima organizacional, en la estructura se observa un alfa de 0,6913, en la comunicación 0,7384, en el liderazgo 0,8132 y en las herramientas motivacionales de 0,7594. En el compromiso calculado arrojó un alfa de 0,694, en el compromiso normativo 0,7094, en el compromiso afectivo de 0,7982 y un alfa de 0,8529 para la totalidad del instrumento.

\section{RESULTADOS}

En las tabla 2 y 3 figuran las puntuaciones medias, las desviaciones típicas y las correlaciones de cada una de las dimensiones de clima organizacional y compromiso organizacional obtenidas con el grupo de empleados de las cinco instituciones de educación superior en Puerto Vallarta.

Las personas encuestadas en las instituciones estudiadas reportan una estructura organizacional favorable, esquemas de comunicación y herramientas motivacionales también adecuados y estilos de dirección altamente favorables. Sin embargo, no se observan los mismos niveles de compromiso; el compromiso calculado y el normativo se reportan como medios y el compromiso afectivo como medio alto.

Los empleados valoran positivamente el clima organizacional sobre todo en las dimensiones de dirección y estructura, donde resaltan espacios adecuados, una permanencia que depende del desempeño personal y no de las preferencias del jefe, aceptación por parte del grupo de trabajo y educación en al trato respecto de los jefes inmediatos. Por lo que respecta al compromiso los trabajadores se sienten medianamente comprometidos con su institución.

Considerados globalmente, los resultados anteriores parecen mostrar que, por lo que se refiere al clima organizacional los empleados perciben que la dirección los apoya y les estimula a con- 
seguir un trabajo en equipo y apoyarse entre sí, permitiéndoles al mismo tiempo cierto grado de autonomía y auto organización; bajo normas claras de actuación y la admisión de nuevas propuestas.

Con respecto al compromiso, en términos generales las evaluaciones que ponderaron al compromiso individual manifiestan colaboradores medianamente comprometidos, aparentemente por un cierto grado de insatisfacción con el papel que cada uno de ellos percibe en relación con las aportaciones al trabajo realizado. Esa falta de reconocimiento no impacta directamente en la forma en que cada directivo manifiesta su estilo de liderazgo, sino que es parte complementaria de la percepción de participación que los colaboradores detectan respecto a su entorno laboral.
Las correlaciones entre las dimensiones de clima organizacional y las del compromiso organizacional que figuran en la tabla 3 muestran que han resultado significativas estadísticamente las relaciones observadas entre las diadas estructura-compromiso normativo, estructura-compromiso afectivo, comunicación-compromiso normativo, comunicacióncompromiso afectivo, motivación-compromiso normativo, motivación-compromiso afectivo y liderazgo-compromiso afectivo. Los resultados sugieren que el clima organizacional y el compromiso son dos variables complejas con cierta dependencia una de la otra, que mantienen relaciones entre sí de moderada dimensión.

De los 12 binomios en que se agrupan las relaciones entre el clima organizacional y el com-

TABLA 2

\section{ESTADÍSTICA DESCRIPTIVA}

\begin{tabular}{|l|c|c|c|c|c|}
\hline & N & MíNIMO & MÁxIM0 & MEDIA & D.S. \\
\hline Estructura & 384 & 9 & 35 & 25,9259 & 5,93630 \\
\hline Comunicación & 384 & 5 & 35 & 25,0741 & 6,58143 \\
\hline Motivación & 384 & 5 & 35 & 25,3889 & 6,57157 \\
\hline Liderazgo & 384 & 5 & 35 & 27,9444 & 7,35980 \\
\hline Compromiso Calculado & 384 & 8 & 35 & 20,963 & 6,09349 \\
\hline Compromiso Normativo & 384 & 9 & 35 & 21,1111 & 5,51727 \\
\hline Compromiso Afectivo & 384 & 10 & 35 & 25,6481 & 5,60376 \\
\hline Clima organizacional & 384 & 40 & 140 & 104,3333 & 21,8865 \\
\hline COMPROMISO & 384 & 34 & 96 & 67,7222 & 12,68994 \\
\hline VÁLlDOS & 384 & & & & \\
\hline
\end{tabular}

Fuente: elaboración propia con el programa estadístico SPSS. 
TABLA 3

\section{CORRELACIONES ENTRE EL CLIMA \\ Y EL COMPROMISO ORGANIZACIONAL}

\begin{tabular}{|c|c|c|c|c|c|}
\hline & & \multicolumn{4}{|c|}{ COMPROMISO ORGANIZACIONAL } \\
\hline & & CÁLCULO & NORMATIVO & AFECTIVO & TOTAL \\
\hline \multirow{5}{*}{$\begin{array}{c}\text { CLIMA } \\
\text { ORGANIZACIONAL }\end{array}$} & Estructura & 0,060 & $0,335^{*}$ & $0,649^{* *}$ & $0,461^{* *}$ \\
\hline & Comunicación & 0,081 & $0,319^{*}$ & $0,582^{* *}$ & $0,435^{* *}$ \\
\hline & Motivación & 0,194 & $0,448^{* *}$ & $0,789^{* *}$ & $0,636^{* *}$ \\
\hline & Liderazgo & 0,056 & 0,024 & $0,414^{* *}$ & 0,220 \\
\hline & TOTAL & 0,118 & $0,329^{*}$ & $0,727^{* *}$ & $0,521^{* *}$ \\
\hline
\end{tabular}

Fuente: Elaboración propia con el programa estadístico SPSS.

Nota. **Correlación significativa al nivel 0,01 .

*Correlación significativa al nivel 0,05.

promiso, correlacionan significativamente siete de ellos que significan $58 \%$ de las posibles combinaciones. Las dimensiones se ordenan según su importancia por la relación mostrada, en primer lugar y con la relación más alta se encuentran las percepciones que se tiene de las herramientas motivacionales y el compromiso afectivo con 0,789, luego la estructura con compromiso afectivo 0,649, le sigue la comunicación con 0,582, las herramientas motivacionales y el compromiso normativo con 0,448 , la estructura y el normativo con 0,335 y por último la comunicación con 0,319 con el compromiso normativo. El resto de las variables no se relacionan significativamente.

Con respecto de las dimensiones del clima organizacional y la medida total de compromiso, correlacionan significativamente al nivel 0,05, la motivación, la estructura y la comunicación con $0,636,0,461$ y 0,435 respectivamente.
Tales resultados sugieren que cuanto más positivo se percibe el factor de motivación, estructura y comunicación del clima organizacional, mayor será el compromiso en su parte afectiva y normativa.

Por otro lado, se observa que básicamente compromiso y clima son dos variables que pueden representar a una empresa que muestre un clima organizacional positivo y, al mismo tiempo una buena parte de sus empleados manifieste cierto grado de lealtad hacia la institución donde trabajan.

\section{CONCLUSIONES}

El estudio se realizó con base en un análisis de las relaciones existentes entre el clima organizacional y el compromiso organizacional en las instituciones de educación superior de Puerto Vallarta. Se analizaron las dimensiones de es- 
tructura, comunicación, liderazgo y motivación, con el fin de relacionar las dimensiones de compromiso afectivo, el compromiso calculado, el compromiso normativo y la posibilidad de intervenirlas con estrategias que las modificaran para incrementar la información que pudiera resultar en estrategias para mejorar la competitividad de cada una.

La investigación se desarrolló en momentos en los que el sistema educativo nacional está viviendo una serie de reformas y modificaciones provenientes de la administración ejecutiva del gobierno federal, dentro de las cuales destacan propuestas y modificaciones de los modelos educativos hacia nuevas estructuras de educación que deberán de implementarse en todos los niveles escolares y cuya asimilación inmediata recae en la estructura administrativa de cada una de las instituciones de educación.

Las conclusiones de la presente investigación se derivan de los resultados obtenidos mediante un análisis de las relaciones con la técnica de correlación estadística, utilizando el coeficiente de correlación de Pearson que explica los efectos que produce el clima organizacional y sus dimensiones en el compromiso, dejando de lado la percepción de los directivos, para medir lo que las personas piensan.

Los datos empíricos obtenidos permiten desplegar alternativas de intervención en instituciones de educación superior, con la intención de generar un clima organizacional idóneo para el incremento continuo del compromiso, originando estándares que le permitan seguir compitiendo en el mercado internacional y nacional.

Según la fuerza y dirección que muestran las relaciones de las variables se planteó a partir de los supuestos teóricos (Hair, Anderson,
Tatham, Black, 2007), y al contrastarse se observó una relación entre la importancia relativa que los colaboradores administrativos de las IES otorgan al clima organizacional y sus niveles de compromiso. Estos hallazgos demuestran que quienes trabajan en las áreas administrativas de las instituciones de educación superior tienen una percepción favorable del clima organizacional, así como un mayor compromiso.

Lo anterior abre el paso a una serie de propuestas de intervención para las instituciones de educación superior. La creación de un clima sano y motivador es básicamente el resultado del comportamiento y el estilo de liderazgo. El clima interno de una organización incluye la naturaleza de las redes de comunicación de las organizaciones, los sistemas de recompensas, el estilo de liderazgo, las técnicas para la fijación de objetivos y otras herramientas.

Al preguntarnos acerca del clima de una organización, lo que realmente queremos saber es lo efectiva que es al movilizar sus recursos humanos por el conjunto de elementos que le rodean y no solo por el intercambio económico generado para obligar un compromiso (Kanter, 1968). El clima es el punto focal de un complejo conjunto de fuerzas en una organización que incide sobre quienes trabajan para ella. Por tanto, conocer las variables del clima permite a los administradores aprovechar esas fuerzas para el logro de las metas organizacionales.

Cuando el clima organizacional es rígido y desequilibrado, es decir, cuando la organización no guarda coherencia entre su sistema de creencias y sus operaciones desencadena, en los grupos que la integran, actitudes de conformidad, de auto-protección o conductas negativas como el ausentismo, apatía, intrigas, robos, conspiraciones y por ende bajo compromiso hacia la institución. 
Sin embargo, las apreciaciones anteriores no deben manejarse como absolutos, dado que los modelos no son únicos y que existen variables adicionales a las observadas. La estrategia de investigación fue confirmatoria de las relaciones existentes entre las dimensiones del clima organizacional y el compromiso organizacional. Sin embargo, no deja de ser una apreciación aislada con una significación estadística que solo ha confirmado la relación entre las variables y sus formas, que además obvia un conjunto de variables que conformarían distintos escenarios, en la medida en que se incrementen en los estudios, mediante modelos alternativos donde se incluyan los efectos de la tecnología en el compromiso (efecto común en las IES), la eficacia de la administración en el compromiso, o se cambie la dependencia e independencia de las variables en los estudios; todo lo anterior con el propósito de continuar enriqueciendo nuestro conocimiento sobre el fenómeno.

\section{Referencias}

ANUIES. (s.f.). Programas nacionales para el mejoramiento de las funciones de la educacion superior. Obtenido de http://www.anuies.mx/servicios/p_anuies/publicaciones/revsup/res073/txt14.htm\#top

Bayona, C., Goñi, S., y Madorran, C. (2000). Compromiso organizacional: implicaciones para la gestión estratégica de los recursos humanos. www.ipna.es .

Betanzos Díaz, N., y Paz Rodríguez, F. (2007). Análisis Psicométrico del compromiso organizacional como variable actitudinal. Anales de Psicología Vol. 23 No. 2 , 207215.

Brunet, L. (2004). El Clima de Trabajo en las Organizaciones: Definicion Diagnostica y Consecuencias. Mexico: Trillas.

Buchanan, B. (1974). Building organizational commitment: The socialization of managers in work organizations. Administrative science quarterly Vol. 19 No.4 , 533-546.
Chiavenato, I., y Colbs. (2001). Administración de Recursos Humanos. Mexico: McGraw Hill.

Denison, D. R. (1991). Cultura Corporativa y Productividad Organizacional. Colombia: Legis, Fondo Editorial.

Domínguez, L. R., Sánchez, J. A., y Torres, Z. (2010). Modelo de ecuaciones estructurales para las relaciones entre el clima organizacional y la productividad. Investigación y Ciencia , 24-32.

Etzioni, A. (1975). A Comparative Analysis of complex organizations. New York: Free Press.

Gairin, J. (1996). La organización Escolar: contexto y texto de actuación. España: La Muralla.

Goncalves, A. (2000). Fundamentos del clima organizacional. Sociedad Latinoamericana para la Calidad.

Hair, J. F., Anderson, R. E., Tatham, R. L., y Black, W. C. (2007). Análisis Multivariante 5a. Ed. Madrid, España: Prentice Hall.

Hall, R. (1996). Organizaciones, estructuras, procesos y resultados. México: Prentice Hall Hispanoamerica S. A. de C. V.

Kanter, R. M. (1968). Commitment and social organization: a study of commitment mechanisms in utopiancommunities. American sociologycal review , 499-517.

Luthans, F. (2008). Comportamiento Organizacional 11 a Edicion. Mexico: McGraw Hill.

Martin, M., y Colbs. (1999). Clima de Trabajo y Eficiencia de centros Docentes: Relaciones Causales. España: Universidad de Alcala.

Marúm-Espinoza, E. (1 de Julio de 2009). www.riseu.unam. $m x$. Recuperado el 15 de Marzo de 2012, de www.riseu.unam.mx: http://www.riseu.unam.mx/documentos/acervo_documental/txtid0042.pdf

Meyer, J. P., y Allen, N. J. (1997). Commitment in the workplace: Theory, research and application. California: Sage Publications Inc.

Mowday, R. T., Porter, L. W., y Steers, R. M. (1982). EmployeeOrganization Linkages: The psychology of commitment, absenteeism and turnover. New York: Academic Press. 
RNA Revista Nacional de Administración

Soberanes R., L. T., y De la Fuente I., A. H. (2009). El Clima y el Compromiso Organizacional en las Organizaciones. Revista Internacional La Nueva Gestión Organizacional Año 5 No. 9 Julio-Diciembre, 120-127.

Tuiran, R. (13 de Octubre de 2011). www.ses.sep.gob.mx. Recuperado el 15 de Marzo de 2012, de www.ses.sep. gob.mx: http://www.ses.sep.gob.mx/work/sites/ses/ resources/PDFContent/2385/13oct11Educacion\%20 Superior\%20en\%20Cifras.pdf

Recibido: 3 de febrero de 2013

Aceptado: 1 de abril de 2013 